\title{
Review
}

\section{Management of Sepsis-Induced Acute Kidney Injury}

\author{
Senaka Rajapakse ${ }^{1}$, Chaturaka Rodrigo ${ }^{2}$, Eranga Wijewickrema ${ }^{2}$ \\ 1Department of Clinical Medicine, Faculty of Medicine, University of Colombo, Sri Lanka \\ 2University Medical Unit, National Hospital, Colombo, Sri Lanka
}

\begin{abstract}
Acute kidney injury (AKI) occurs in a significant proportion of patients with severe sepsis, and is an important cause of mortality in such patients. Current concepts of pathogenesis of AKI are shifting from vasoconstriction-ischaemia induced injury to toxic and immune mediated injury and hyperaemic injury resulting in apoptosis of renal cells. Renal replacement therapy is the mainstay of management of AKI. Adequacy of dialysis is likely to be linked to better outcome, but there is still no clear consensus on the timing, modality, intensity or frequency of dialysis. Haemodynamically unstable patients usually require modes of continuous renal replacement therapy. Biocompatible dialyser membranes are likely to be safer than older cellulose membranes. Bicarbonate is preferred to acetate and lactate as dialysate buffer. Anticoagulation has to be undertaken with care to prevent excessive haemorrhage in the setting of already deranged haemostasis. Adequate volume resuscitation and maintenance of renal perfusion by the use of vasopressors is beneficial; norepinephrine is the vasopressor of choice. There is no place for the use of low- or renal-dose dopamine, mannitol or frusemide in the setting of sepsisinduced AKI, and in fact they may be detrimental. Prevention of kidney damage by nephrotoxic drugs and radio-contrast media is of vital importance. Careful dose management of nephrotoxic drugs will prevent renal injury. Hydration prior to administration of contrast media prevents nephrotoxicity, but the benefit of $\mathrm{N}$-Acetylcysteine is unclear. Tight glycaemic control may have renoprotective effects, though its place in the management of severe sepsis is now controversial. No clear evidence of benefit is seen with other newer therapies.
\end{abstract}

\section{Introduction}

Deterioration of renal function over a short period is termed acute kidney injury (AKI). AKI has replaced the term acute renal failure, and is defined according the RIFLE criteria ${ }^{1}$. Sepsis is the presence of SIRS with infection. When acute kidney injury occurs in the presence of sepsis, without other clear and established non-sepsis related causes of AKI, it is considered sepsisinduced. AKI affects approximately $35 \%$ of intensive care unit (ICU) patients ${ }^{1}$, and around $50 \%$ of these are due to sepsis ${ }^{2}$. While AKI has an overall mortality rate of $45 \%$; the mortality rate of sepsis-induced AKI is much higher, at over $70 \%{ }^{1,3}$. The severity of AKI positively correlates with morbidity and mortality of ICU patients. A linear relationship has been demonstrated between the stage of AKI stage and mortality ${ }^{3}$.
A clear understanding of pathophysiology, prevention and treatment of sepsis-induced AKI is essential for critical care physicians. We review the evidence base for aspects of renal replacement therapy, supportive therapies, and preventive aspects in sepsisinduced AKI.

\section{Pathophysiology of sepsis-induced AKI}

The pathophysiology of sepsis induced AKI is poorly understood. Traditionally, AKI in sepsis and septic shock is thought to result from renal ischaemia secondary to inadequate renal blood flow (RBF).

Correspondence: Senaka Rajapakse Faculty of Medicine, University of Colombo, 25, Kynsey Road, Colombo 08, Sri Lanka. Email: senaka.ucfm@gmail.com 
This construct implies that restoration of RBF before acute tubular necrosis or cortical necrosis occurs should be the primary means of renal protection in septic patients with the risk of AKI. However, recent studies have shown that the renal circulation participates in the systemic vasodilatation observed during severe sepsis/septic shock, and the development of septic AKI occurs in the setting of adequate and even increased renal perfusion rather than renal hypoperfusion ${ }^{4,5}$. Thus, sepsisinduced AKI may represent a unique form of AKI, namely hyperaemic AKI. Microcirculatory changes which typically occur in other tissues in severe sepsis also occur in the kidney, resulting in tissue hypoperfusion despite normal macrovascular blood flow. It has been suggested that internal redistribution of blood flow occurs in sepsis, favouring the cortex and leading to medullary ischaemia and acute tubular damage. Nonetheless, in animal models, cortical and medullary blood flow measured by laser Doppler flowmetry remains unchanged in sepsis with hyperdynamic circulation ${ }^{6}$. The paucity of histological evidence from patients with sepsis-induced AKI is one reason for the poor understanding of its pathogenesis; most of our evidence is from surrogate markers of kidney damage, such as urine output, urinary sodium concentration, fractional excretion of sodium and fractional excretion of urea.

Toxic and immunologic mechanisms are clearly important in mediating renal injury during sepsis. This is due to the release of a vast array of inflammatory cytokines, arachidonate metabolites, vasoactive substances, thrombogenic agents, and other biologically active mediators. Tumour necrosis factor- $\alpha$ (TNF$\alpha$ ) has been demonstrated to play a major role in pathogenesis of AKI in gramnegative septic shock ${ }^{7}$, through direct effects on glomerular endothelial and tubular cells resulting in apoptosis. Thus, the paradigms currently used to explain AKI in sepsis is shifting from vasoconstriction and ischaemia to vasodilatation and hyperaemia, and from acute tubular necrosis to acute tubular apoptosis.

\section{Modes of renal replacement therapy}

Modalities of renal replacement therapy

Renal replacement therapy is the mainstay of sepsis-induced AKI. While many modalities of renal replacement therapy are available, they all rely on two basic principles - diffusion and convection ${ }^{8}$. Dialysis depends primarily on diffusion, while haemofiltration uses convection. The two modalities may be combined.

\section{Diffusion methods}

The two diffusion methods used are peritoneal dialysis and intermittent haemodialysis. Peritoneal dialysis, where the peritoneum acts as the semipermeable membrane, is rarely used in critically ill patients now. In this method, a peritoneal catheter is introduced through the anterior abdominal wall into the peritoneal cavity and a dialysis solution is cyclically introduced into and drained out from the peritoneal cavity. Urea, creatinine and other undesired molecules diffuse down their concentration gradients, and the solutes in the dialysate will readjust the constituents of plasma. If fluid removal is required, glucose is added to the dialysate to create ultrafiltration of water. An open randomised trial comparing peritoneal dialysis vs. haemofiltration in sepsis induced acute renal failure showed that peritoneal dialysis was both inferior as well as more expensive than haemofiltration ${ }^{9}$.

Intermittent haemodialysis is used in haemodynamically stable patients with ARF. Heparinized venous blood from the patient is made to pass through an extracorporeal dialyser, which seperates it from a crystalloid solution by a semipermeable membrane. Urea, creatinine and other toxins pass down their chemical gradients in to the dialysis solution while the reverse process restores the natural composition of plasma. By adjusting a pressure difference on either side of the membrane an osmotic drag can be created for water molecules to pass down in to the dialysate, resulting in a net loss of water: this process, known as ultrafiltration, is used to remove fluid in fluid overloaded states. The major disadvantage of dialysis is that although it removes smaller molecules 
such as urea efficiently, with 'middle' size (creatinine) and larger molecule the clearance can be quite unsatisfactory(8).

\section{Convective methods}

Haemofiltration is the commonly used convective modality. Blood is passed through a dialyser where it is separated from a crystalloid solution by a semipermeable membrane. A hydrostatic pressure is created on that side of the membrane to filter out most of the smaller and middle size molecules. This ultrafiltrate is discarded and replaced with a replacement fluid of desirable concentrations of solutes. Volume is managed by adjusting the volume of replacement fluid. Two methods of haemofiltration are used- continuous arteriovenous haemofiltration $(\mathrm{CAVH})$ and continuous venovenous haemofiltration (CVVH). In CAVH the femoral artery is cannulated and blood is sent through an extracorporeal circuit. The arterial pressure drives the ultrafiltrate through the membrane. Though the design is simple, the method has the risk of a simple breach in the closed circuit resulting in exsanguination. Furthermore, the ultrafiltration rates achieved by arterial pressure alone are not adequate to clear solutes adequately as demanded, and clearance rates are even lower in hypotensive patients. Slow blood flow can result in frequent clotting of the extracorporeal circuit. In CVVH a vein (usually the femoral or internal jugular vein) is accessed. The venous blood is sent through an occlusive pump, which gives better control over filtration; and rates over $100 \mathrm{ml} / \mathrm{min}$ can be achieved. CVVH is currently the preferred modality of renal replacement therapy in critically ill patients, especially when they are haemodynamically unstable.

\section{The role of renal replacement therapy (RRT) in acute kidney injury}

The key issues in the use of renal replacement therapy are whether the modality, timing, and frequency of RRT influence mortality. These aspects are discussed in detail below.

\section{Modality of dialysis}

Continuous renal replacement therapy (CRRT) has an advantage over intermittent haemodialysis in that it provides greater haemodynamic stability, easier fluid removal and greater flexibility in providing parenteral nutrition as a result of greater control over fluid balance ${ }^{10,11}$. The disadvantages of continuous therapy are the need for long periods of anticoagulation of the patient to maximize the life span of the filter, complications related to vascular access, and greater economic cost.

Current evidence however, does not demonstrate a significant advantage of CRRT over IHD in terms of mortality or renal recovery. A recent meta-analysis by Bagshaw et al ${ }^{12}$ comparing CRRT with IHD as the initial modality of renal replacement concluded that there was no significant difference in outcome in terms of mortality or renal recovery between the two modalities. It was suggested that CRRT resulted in fewer episodes of haemodynamic imbalance and better fluid balance; nonetheless the relevant studies had numerous flaws with regard to design, data description, conduct and quality. A systematic review Pannu et al ${ }^{13}$ also showed that clinical outcomes with CRRT and IHD were similar. Overall, the current state of evidence does not indicate a clear advantage in the use of CRRT over IHD in sepsis induced ARF, although better quality evidence from large, multicentre trials are required. Given its advantages in patients who are haemodynamically unstable, CRRT is likely to be the more favoured modality where available. In haemodynamically stable patients, there is inadequate justification as yet to favour one modality over the other. In resource limited settings, IHD will remain the main modality of dialysis. Despite peritoneal dialysis being inferior, it may be the only available option in haemodynamically unstable patients in certain resource poor settings.

Whether dialysis or haemofiltration will help in removal of inflammatory mediators and hence help alleviate the cytokine storm in severe sepsis is unknown. Conventional ultrafiltration rates are probably inadequate to have a significant effect on cytokine removal, and for this 
purpose High Volume Haemofiltration (HVHF), using ultrafiltration rates $>35 \mathrm{ml} / \mathrm{kg} / \mathrm{hr}$ have been used. There is some evidence of improved haemodynamics, reduced need for vasopressor support ${ }^{14-16}$ and a trend towards improved survival with HVHF17, but further studies are required. It has been suggested that the improved haemodynamics seen with CRRT may be a result of cytokine removal, and also mild hypothermia resulting in an increase systemic vascular tone.

\section{Timing of RRT}

The specific indications for initiation of RRT in sepsis induced ARF are different from those of chronic renal failure. The traditional indications seen in chronic renal failure such as encephalopathy and pericarditis are less common in AKI; also, these clinical features may be due to numerous other reasons in critical illness. Incipient or established fluid overload, electrolyte imbalance and acidosis which do not respond adequately to medical management are clear indications for initiation of RRT. Evidence regarding the ideal timing of dialysis is scarce. Three RCTs ${ }^{18-20}$ have shown inconclusive results. A prospective cohort study ${ }^{21}$ showed that the risk of death was lower when dialysis was started at lower blood urea levels. A specific blood urea nitrogen or creatinine level at which RRT should be commenced in ARF is difficult to define, and no firm recommendation can be made based on current evidence as to the ideal timing of initiation of RRT. Direct clinical or biochemical indications for RRT will guide the initiation of RRT in sepsis induced ARF. However, given the limited evidence of possible benefit in early initiation of RRT, it would seem logical to initiate RRT early rather than late, especially in rapidly developing, symptomatic, oliguric renal failure with metabolic derangement.

\section{Frequency and intensity of RRT}

The use of urea clearance alone as a measure of adequacy of dialysis has been questioned at recent times, as many other small and middle sized molecules contribute to the adverse effects of AKI.
This argument is further strengthened by the fact that in addition to urea and creatinine there may be many other 'small' and middle' size molecules that may contribute to the symptomatology of chronic renal failure. A Kt/V value of greater than 1 has been traditionally accepted as a measure of adequate dialysis in chronic renal failure. However, the high catabolic rate in sepsis induced ARF, variable fluid volumes, and the post-dialysis 'rebound' of urea concentrations from hypoperfused organs, limits the use of Kt/V as a measure of adequacy of dialysis in critically ill patients. The 'dose' of renal replacement therapy in IHD in acute renal failure is often described using the hours of dialysis, rate of blood flow, and frequency of dialysis. Since urea equilibrates rapidly across the dialysis membrane, urea clearance in CRRT is essentially equivalent to the volume of effluent dialysate (including any ultrafiltered fluid), and therefore CRRT dose is commonly expressed as $\mathrm{L} / \mathrm{kg}$ per hour of effluent.

Gillum et al 22 prospectively studied the effect of dialysis intensity on survival in patients with ARF. The trial compared patients receiving intensive intermittent dialysis (daily dialysis with 5 -6 hours per treatment) and the other group had standard non-intensive prescription of 5 hour treatments from a daily basis to every third day basis. The survival in two groups did not vary significantly. The technical capacity of modern dialysers have increased significantly since then, and these findings have been challenged. Schiffl et $\mathrm{al}^{23}$ in another trial demonstrated a higher survival among patients with ARF treated with daily IHD compared with those treated with thrice weekly IHD; the dose of dialysis in the conventional group was, however, lower than that recommended in chronic renal failure. In this study, patients needing CRRT were excluded, possibly resulting in exclusion of severely ill patients; hence the patient population may not be truly representative of the range of patients with sepsis induced AKI, many of whom would be in septic shock, needing CRRT. To further support this, the overall mortality of the study was $37 \%$, which is much lower 
than standard mortality rates for sepsis induced AKI.

Ronco et $\mathrm{al}^{24}$ evaluated the dose effect on outcome of patients treated with CVVH. They randomized patients to three groups and prescribed three ultra filtration rates based on body weights for each group. $(20,35,45 \mathrm{ml} / \mathrm{hr} / \mathrm{kg})$. A significant survival benefit was seen in patients who received middle and high ultrafiltration rates vs. the low filtration rate group. Thus it appears that a minimum ultrafiltration rate of $35 \mathrm{ml} / \mathrm{kg} / \mathrm{h}$ is required to have a survival benefit. Significantly, this study highlighted the inadequacy of routine CVVH prescriptions and ultrafiltration rates used in most centers. Based on these findings, for successful haemofiltration the filtration fraction (ultrafiltration rate / blood flow rate) has to be around $30-35 \%$, and to achieve an ultrafiltration rate of 35 $\mathrm{ml} / \mathrm{kg} / \mathrm{h}$, a blood flow of at least 250 $\mathrm{ml} / \mathrm{min}$ is necessary as opposed to the current rate around $150 \mathrm{ml} / \mathrm{min}$ used in most centres.

Contradicting this, results from the recent ATN trial do not support these findings, concluding that intensive RRT does not improve mortality or hasten recovery compared with non-intensive dialysis ${ }^{25}$. Overall, evidence regarding the ideal dose of renal replacement therapy in sepsis induced ARF is inadequate to make firm recommendations, and larger welldesigned RCTs are required.

Other aspects related to RRT which must be considered are the type of semipermeable membrane used in the dialyser, and the type of buffer used, and issues related to anticoagulation. The semipermeable membrane that separates the body fluids and the crystalloid solution in the dialyser influences the efficiency of the procedure. Membranes are of two types- biocompatible membranes and others. Non-biocompatible membranes are thought to cause activation of inflammatory cells and thus the release of inflammatory mediators, resulting in bronchoconstriction, vasoconstriction and hypotension, which are clearly undesirable in the setting of sepsis.

Definite evidence that the possible pro-inflammatory effects has deleterious effects in septic patients is not available. Early cellulose based versions of dialysis membranes were bio-incompatible, but modification of the cellulose component has made it possible to create synthetic polymers that are biocompatible. Biocompatible membranes may have the added advantage of adsorbing mediators involved in the sepsis cascade. Evidence from several RCTs ${ }^{26-29}$ suggests that the use of non-biocompatible membranes is associated with an increased risk of death; hence their use is not recommended in acute renal failure. On the downside, synthetic membranes are more expensive.

Dialysate contains a buffer to correct metabolic acidosis - acetate, lactate and bicarbonate are used. In septic patients, the inadequacy of organs to convert lactate or acetate to bicarbonate is a potential issue -hence bicarbonate buffer is preferred on theoretical grounds. A comparison of bicarbonate and lactate in patients receiving CRRT showed no difference in survival, although cardiovascular events were significantly less with bicarbonate ${ }^{30}$. Overall, however, there is no evidence that bicarbonate is superior to acetate or lactate $^{31}$, or that there is any difference between acetate and lactate ${ }^{32}$, although evidence is only from small studies.

Anticoagulation is required in both IHD and CRRT to prevent clotting of the filter in the extracorporeal circuit, and this is of particular importance in CRRT. Because of the coagulopathy which frequently occurs in severe sepsis, anticoagulation can result in serious bleeding.

Unfractionated heparin is most commonly used, although the optimal intensity of anticoagulation is not known. Fractionated heparin is not used because of the difficulties in reversing its effects. In patients at high risk of bleeding saline flushes or citrate infusion are used. There is some evidence that citrate reduces the risk of bleeding compared to heparin ${ }^{33}$, although it could cause hypocalcaemia, metabolic alkalosis and citrate toxicity. Hirudin maybe an alternative to heparin, but data is inadequate at present. 


\section{Non-dialytic strategies for preventing and treating sepsis induced acute kidney injury}

While clearly dialysis improves outcome in sepsis induced AKI, many other strategies are also of value. In particular, careful attention to hydration and volume loading, maintenance of mean arterial pressure using vasopressors and inotropes, and avoidance of ionic contrast agents and nephro-toxic drugs are of benefit.

\section{Hydration and volume loading}

Although no randomized controlled trials (RCTs) have been carried out, it has long been recognized that intravascular volume depletion is an important risk factor for development of AKI and its correction with fluids lead to resolution of AKI. Rivers et $\mathrm{al}^{34}$ demonstrated that early aggressive resuscitation reduced mortality in septic shock, although direct evidence of prevention of AKI was not documented in the trial. In certain settings, such as rhabdomyolysis, early and aggressive fluid resuscitation has clearly been shown to be beneficial ${ }^{35}$.

\section{Maintaining renal perfusion pressure}

While clearly maintaining adequate renal perfusion is of paramount importance, recommendations are based more on expert opinion than trial evidence. In general, a mean arterial pressure above $65 \mathrm{mmHg}$ is considered adequate; however this value is arbitrary, and targets should be based on the physiological state of the patient, and other outcome measures such as urine output, measures of intravascular volume adequacy, and biochemical values.

The profound vasodilation which occurs in severe sepsis results in resistant shock and reduction in renal perfusion. Vasopressor agents-such as, high-dose dopamine, epinephrine, phenylephrine, or low dose vasopressin or terlipressin- can be used to restore an acceptable mean arterial blood pressure, once adequate volume repletion is accomplished. Despite previous fears that vasopressors may result in further renal vasoconstriction and reduced renal perfusion, evidence has been to the contrary- renal perfusion improves significantly with norepinephrine in patients with septic shock ${ }^{36}$. This probably occurs partly due to the rise in mean arterial pressure and partly due to the renal vasodilatation caused by decreased renal sympathetic tone through baroreceptor stimulation by increase in systemic blood pressure $^{37}$. Norepinephrine is superior to high-dose dopamine in restoring target blood pressure in septic shock, and is associated with lower mortality compared to other vasopressor agents ${ }^{38}$. Norepinephrine is currently the vasopressor of choice in septic shock. Phenylephrine and adrenaline are not recommended as first line agents because of concern regarding unbalanced vasoconstriction with phenylephrine, together with lack of sufficient human data, and in the case of adrenaline, concern about its greater tendency to induce hyperlactaemia, acidosis, hyperglycaemia and tachycardia. On the other hand, lowdose vasopressin, when used in combination with norepinephrine, allowed decreasing the dose of norepinephrine in the treatment of septic shock without demonstrating any other added benefit ${ }^{39}$.

Increased intra-abdominal pressure results in reduced renal perfusion. Prompt recognition and early surgical treatment of increased intra-abdominal pressure is often neglected, but of utmost importance ${ }^{40}$.

\section{Low-dose dopamine}

In healthy subjects, low-dose dopamine 0.5 to $3 \mu \mathrm{g} / \mathrm{kg} / \mathrm{min}$ ) increases renal blood flow and promotes natriuresis through stimulation of renal D1, D2 and D4 receptors ${ }^{41}$. Based on this, so called lowdose dopamine or renal-dose dopamine has long been touted as being renoprotective. 42,43. However, repeated studies failed to demonstrate this benefit, and a recent metaanalysis showed no significant benefit in the use of low-dose dopamine, in reducing death or need for renal replacement therapy ${ }^{44}$. Low-dose or renal-dose has no place in the management of critically ill patients. This recommendation does not preclude the use of dopamine in higher, 'inotropic' doses. 


\section{Drug induced nephrotoxocity}

Many patients with sepsis are critically ill often necessitating the use of multiple therapeutic agents, many of which may individually or in combination have the potential to cause kidney injury. Nephrotoxic drugs maybe a contributing factor in $19 \%$ to $25 \%$ of cases of severe acute renal failure in critically ill patients ${ }^{3,45}$. Nephrotoxicity occurs through various mechanisms.

Aminoglycosides, vancomycin and amphotericin B are the most commonly used drugs in septic patients which cause acute tubular necrosis (ATN). Toxicity results from sustained elevations of drug levels, that occur from multiple daily doses. Aminoglycosides show peak dependent bactericidal effects, and hence once daily dosing has been shown to minimise this effect without any effect on efficacy ${ }^{46-48}$. Vancomycin results in nephrotoxicity in 6\% to $30 \%$ of patients ${ }^{49}$. Both peak and trough levels contribute to toxicity, and careful consideration of renal function, and monitoring of trough levels are essential with its use. Amphotericin B associated nephrotoxicity occurs in $25-30 \%$ of patients, with progressive increase in the risk of AKI with increase in cumulative dose 50. The risk of renal dysfunction is relatively low at doses of $<0.5 \mathrm{mg} \mathrm{kg}^{-1}$.day and a cumulative dose of $<600 \mathrm{mg}$. The use of lipid formulations of amphotericin B seems to cause less nephrotoxicity compared with standard formulations ${ }^{51,52}$.

\section{Drug induced acute interstitial nephritis}

Many drugs, which are commonly used in the critical care setting, are associated with acute interstitial nephritis (NSAIDs, betalactams, sulphonamides, loop diuretics, thiazides, quinolones, cimetidine, allopurinol, proton pump inhibitors), and account for $3 \%$ to $15 \%$ of all drug induced acute renal failure ${ }^{53}$. Renal dysfunction usually occur 7-14 days after exposure and when it occurs secondary to $\beta$-lactam antibiotics and sulfa drugs, may be associated with fever, eosinophilia and rash. Renal manifestations include sterile pyuria, eosinophiluria, and an inflammatory infiltrate in renal interstitium on the biopsy. Reactions are generally idiosyncratic, and management involves removal of the suspected causative agent and supportive therapy. Treatment with prednisolone $1 \mathrm{mg} / \mathrm{kg} /$ day for up to 4 weeks may accelerate the rate of recovery 53,54 .

\section{Radiocontrast nephrotoxicity}

Iodinated contrast media are commonly used during the diagnostic workup of critically ill patients. AKI is a well recognized complication of contrast media resulting in increased in hospital mortality, prolonged hospital stay and increased health care costs 55,56 . The most important risk factor for developing AKI following contrast administration is the baseline glomerular filtration rate (GFR), with increased risk of AKI below estimated GFR of $60 \mathrm{ml} / \mathrm{min}$. Other risk factors include diabetes mellitus, heart failure, volume depletion, nephrotoxic drugs, and haemodynamic instability.

The volume and type of contrast media administered influence the risk of contrast nephropathy in critically ill patients. The volume of contrast medium administered correlates with nephrotoxicity 57-59. The use of isosmolar (approximately $290 \mathrm{mOsm} / \mathrm{kg}$ ) contrast media is associated with considerably less nephrotoxicity compared to low (500-800 $\mathrm{mOsm} / \mathrm{kg}$ ) and high osmolar contrast media60,61. Volume expansion prior to administration of contrast, preferably with isotonic fluids prevents contrast nephropathy. Intravenous fluid administration is likely to be preferable to oral hydration ${ }^{62}$. Isotonic fluids, (in particular normal saline) are preferable to hypotonic fluid resuscitation in the prevention of $\mathrm{AKI}^{63}$.

Although $\mathrm{N}$-acetylcysteine (NAC) has been widely suggested to be of benefit, its beneficial effects have not been consistently seen. A recent meta-analyses showed that significant heterogeneity in NAC effect existed across studies, and that overall, no benefit was seen ${ }^{64}$. NAC is known to reduce serum creatinine (by activating creatinine kinase activity and possibly reducing tubular secretion) without improving GFR, and this effect may confound the results of clinical trials of its use in preventing contrast induced kidney injury ${ }^{65}$. The value of NAC in preventing 
contrast nephropathy clearly needs further study, in view of the wide heterogeneity of effect shown in the different studies ${ }^{64}$. However given its relatively low incidence of side effects and low cost, together with evidence of benefit, current practice favours its use in addition to hydration in high risk patients receiving contrast.

Other pharmacologic agents suggested to be of benefit include theophylline, statins, ascorbic acid, and prostaglandin E1. Fenoldapam, dopamine, calcium channel blockers, atrial natriuretic peptide, and L-arginine have been shown to be ineffective. Theophylline shows some promise ${ }^{66}$. Furosemide, mannitol, and an endothelin-receptor antagonists are potentially detrimental ${ }^{67}$. The place of dialysis needs further study ${ }^{68}$, with no current evidence of preventive benefit in contrast nephropathy.

\section{The role of loop diuretics}

Despite some evidence from in-vitro studies that furosemide causes reduced expression of certain inflammatory mediators, there is no evidence that loop diuretics are of benefit in prevention or treatment of sepsisinduced AKI. Oliguric AKI, is associated with increased mortality compared to nonoliguric AKI ${ }^{69}, 70$. Hence clinicians often use loop diuretics to convert oliguric AKI to non-oliguric $\mathrm{AKI}^{71,72}$. Trial evidence however suggests that furosemide may actually increase the risk of death/ nonrecovery of renal function ${ }^{71,72}$ and two recent systematic reviews and a metaanalysis showed that loop diuretics are of no benefit in AKI73-75.

\section{The role of osmotic diuretics - mannitol}

It has been suggested that mannitol attenuates renal damage in animal models, increases renal blood flow and acts as a free radical scavenger preventing reperfusion injury of the kidney ${ }^{76}$. In practice however, the use of mannitol in patients with mild to moderate renal insufficiency was shown to be associated with greater risk of AKI when compared with saline alone ${ }^{77,78}$. Thus the use of mannitol should be discouraged.

\section{Insulin and tight glycaemic control}

Although tight glycaemic control was thought to reduce mortality in critically ill patients, current evidence is to the contrary- a recent meta-analysis showed a high incidence of hypoglycaemic events, with no evidence of mortality benefit ${ }^{79}$. However, tight glycaemic control using insulin in critically ill patients has been shown to be reno-protective, preventing oliguria and reducing the need for RRT ${ }^{80}$. The anti-inflammatory action of insulin may play a role in this effect. Further evidence is awaited.

\section{Conclusions}

Renal replacement therapy is the mainstay of management of sepsis induced acute renal failure. An adequate dose of dialysis is likely to improve outcome, though currently no consensus is available on the ideal dose, frequency or modality. Prevention of drug induced and contrast induced nephropathy is of great importance. Adequate volume resuscitation and the use of the correct inotrope are also important. Many other therapies have been tried, without clear benefit. Much controversy exists on many of these issues, and current evidence supports the concept that management of sepsis induced AKI should be fine tuned to the individual patient.

\section{References}

1. Ostermann $\mathrm{M}$, Chang RWS. Acute kidney injury in the intensive care unit according to RIFLE. Crit Care Med 2007; 35: 1837-1843

2. Uchino $S$, Kellum JA, Bellemo R, et al. Acute renal failure in critically ill patients: A multinational, multicenter study. Beginning and Ending Supportive Therapy for the Kidney (BEST Kidney) Investigators. JAMA 2005;294:813-818

3. Hoste EA, Clermont G, Kersten A, et al. RIFLE criteria for acute kidney injury is associated with hospital mortality in critical ill patients: A cohort analysis. Critical Care 2006; 10: R73

4. Brenner M, Schaer GL, Mallory DL, et al. Detection of renal blood flow abnormalities in septic and critically ill patients using a newly designed 
indwelling thermodilution renal vein catheter. Chest 1990; 98: 170-179

5. Langenberg C, Bellomo R, May CN. Renal vascular resistance in sepsis. Nephron Physiol 2006; 104: 1-11

6. Di Giantomasso D, Morimatsu H, May $\mathrm{CN}$, et al. Effect of Intra-renal blood flow distribution in hyperdynamic septic shock. Crit Care Med 2003; 31: 25092513

7. Cunningham PN, Dyanov HM, Park P, et al. Acute renal failure in endotoxaemia is caused by TNF acting directly on TNF receptor-1 in kidney. J Immunol 2002; 168: 5817-5823

8. Pastan S, Bailey J. Dialysis therapy N Eng J Med; 1998: 1428 - 37.

9. Phu NH, Hien TT, Mai NT et al. Hemofiltration and peritoneal dialysis in infection-associated acute renal failure in Vietnam. N Engl J Med. 2002; 347: 895-902.

10. Forni LG, Hilton PG. Continuous haemofiltration in treatment of acute renal failure. N Eng J Med 2007; 336: 1303-09.

11. Huang Z, Letteri JJ, Clark WR et al. Operational characteristics of continuous renal replacement modalities used for critically ill patients with acute kidney injury. Int J Artif Organs 2008; 31: 525-34.

12. Bagshaw SM, Berthiaume LR, Delaney A et al. Continuous versus intermittent renal replacement therapy for critically ill patients with acute kidney injury: A meta-analysis. Crit Care Med 2008; 36: 610-7.

13. Pannu N, Klarenbach S, Wiebe N, Manns B, Tonelli M. Renal Replacement Therapy in Patients With Acute Renal Failure. JAMA 2008; 299: 793-805.

14. Cole L, Bellomo R, Journois D et al. Highvolume hemofiltration in human septic shock. Intensive Care Med 2001; 27: 978-86.

15. Honore PM, Jamez J, Wauthier $\mathrm{M}$ et al. Prospective evaluation of short-term, high-volume isovolemic hemofiltration on the hemodynamic course and outcome in patients with intractable circulatory failure resulting from septic shock. Crit Care Med 2000; 28: 35813587
16. Ratanarat $\mathrm{R}$, Brendolan A, Piccinni $\mathrm{P}$ et al. Pulse high volume haemofiltration for treatment of severe sepsis: effects on hemodynamics and survival. Crit Care 2005; 9: R294-R302

17. Oudemans-van Straaten HM, Bosman RJ, van der Spoel JI et al. Outcome of critically ill patients treated with intermittent high-volume hemofiltration: a prospective cohort analysis. Intensive Care Med 1999; 25 : 814-21

18. Ronco C, Bellomo R, Hommel $\mathrm{P}$ et al. Effects of different doses in continuous veno-venous hemofiltration on outcomes in acute renal failure: a prospective, randomized trial. Lancet 2000; 355: 26-30.

19. Bouman CSC, Oudemans-Van Straaten HM, Tijssen JGP et al. Effects of early high-volume continuous venovenous hemofiltration on survival and recovery of renal function in intensive care patients with acute renal failure. Crit Care Med. 2002; 30: 2205-11.

20. Sugahara S, Suzuki H. Early start on continuous hemodialysis therapy improves survival rate in patients with acute renal failure following coronary bypass surgery. Hemodial Int. 2004; 8: 320-325.

21. Liu KD, Himmelfarb J, Paganini E et al. Timing of initiation of dialysis in critically ill patients with acute kidney injury. Clin J Am Soc Nephrol. 2006; 1: 915-19.

22. Gillum DM, Dixon BS, Yanover MJ et al. The role of intensive dialysis in acute renal failure. Clin Nephrol 1986; 25: 249-55

23. Schiffl H, Lang SM, Fischer R. Daily hemodialysis and the outcome of acute renal failure. N Engl J Med 2002; 346: 305-10.

24. Ronco C, Bellomo R, Homel $\mathrm{P}$ et al. Effects of different doses in continuous veno-venous haemofiltration on outcomes of acute renal failure: a prospective randomised trial. Lancet 2000; 356: 26-30

25. The VA/NIH Acute Renal Failure Trial Network. Intensity of renal support in critically ill patients with Acute Kidney Injury. N Engl J Med 2008; 359: 1-20 
26. Gastaldello K, Melot C, Kahn RJ et al. Comparison of cellulose diacetate and polysulfone membranes in the outcome of acute renal failure. Nephrol Dial Transplant 2000; 15: 224-230.

27. Jorres A, Gahl GM, Dobis C et al. International Multicentre Study Group. Haemodialysis-membrane biocompatibility and mortality of patients with dialysis dependent acute renal failure. Lancet 1999; 354: 13371341.

28. Himmelfarb J, Tolkoff RN, Chandran P et al. A multicenter comparison of dialysis membranes in the treatment of acute renal failure requiring dialysis. J Am Soc Nephrol 1998; 9: 257-66.

29. Jones CH, Goutcher E, Newstead CG et al. Hemodynamics and survival of patients with acute renal failure treated by continuous dialysis with two synthetic membranes. Artif Organs 1998; 22: 638643.

30. Barenbrock M, Hausberg M, Matzkies F e al. Effects of bicarbonate-and lactatebuffered replacement fluids on cardiovascular outcome in CVVH patients. Kidney Int 2000; 58: 1751-57

31. Bret M, Hurot JM, Mercatello A et al. Acetate free biofiltration for acute renal failure. Ren Fail 1998; 20: 493-503.

32. Morgera S, Heering P, Szentandrasi T et al. Comparison of a lactate-versus acetate-based hemofiltration replacement fluid in patients with acute renal failure. Ren Fail 1997; 19: 155164.

33. Kutsogiannis DJ, Gibney RTN, Stollery D et al. Regional citrate versus systemic heparin anticoagulation for continuous renal replacement in critically ill patients. Kidney Int 2005; 67: 23612367.

34. Rivers E, Nguyen, B, Havstad S et al. Early Goal-Directed Therapy in the Treatment of Severe Sepsis and Septic Shock. N Engl J Med 2001; 345: 13681377

35. Better OS, Rubinstein I. Management of shock and acute renal failure in casualties suffering from the crush syndrome. Ren Fail 1997;19:647-653

36. Schaer GL, Fink MP, Parrillo JE. Norepinephrine alone versus plus low- dose dopamine: Enhanced renal blood flow with combination pressor therapy. Crit Care Med 1985; 13: 492-496

37. Anderson WP, Komer PI, Selig SE. Mechanisms involved in the renal responses to intravenous and renal artery infusions of noradrenaline in conscious dogs. J Physiol 1981; 321: 2130

38. Martin C, Viviand $\mathrm{X}$, Leone $\mathrm{M}$, et al. Effect of norepinephrine on the outcome of septic shock. Crit Care Med 2000; 28: 2758-2765

39. Russell JA. Vasopressin in vasodilatory and septic shock. Curr Opin Crit Care 2007; 13: 383-391

40. Malbrain ML, Chiumello D, Pelosi P et al. Incidence and prognosis of intraabdominal hypertension in a mixed population of critically ill patients: A multiple- center epidemiological study. Crit Care Med 2005; 33: 315-322

41. Denion MD, Chertow GM, Brady HR. "Renal-dose" for the treatment of acute renal failure: scientific rationale, experimental studies and clinical trials. Kidney Int 1996; 50: 504-514

42. McHugh GJ. Current usage of dopamine in New Zealand intensive care units. Anaesth Intensive Care 2001; 29: 623626

43. Prins I, Plotz FB, Uiterwaal CS et al. Lowdose dopamine in neonatal and pediatric intensive care: a systematic review. Intensive Care Med. 2001; 27: 206-210

44. Friedrich JO, Adhikari N, Herridge MS et al. Meta-Analysis: Low-dose dopamine increases urine output but does not prevent renal dysfunction or death. Ann Intern Med 2005; 142: 510-524

45. Mehta RL, Pascual MT, Soroko S, et al: Spectrum of acute renal failure in the intensice care unit: The PICARD experience. Kidney Int 2004; 66: 16131621

46. Barza M, Ionnidis JP, Cappelleri JC et al. Single or multiple daily doses aminoglycosides: A meta-analysis. BMJ 1996; 312: 338-345

47. Hatala R, Dinh T, Cook DJ. Once daily aminoglycoside dosing in immunocompetent adults: A meta- 
analysis. Ann Intern Med 1996; 124: 717-725

48. Hatala R, Dinh TT, Cook DJ. Single daily dosing of aminoglycosides in immunocompromised adults: A systematic review. Clin Infect Dis 1997; 24: 810-815

49. Hidayat LK, Hsu DI, Quist R et al. High dose vancomycin therapy for methicillin-resistant Staphylococcus aureus infections: Efficacy and toxicity. Arch Intern Med 2006; 166: 2138-2144

50. Habarth S, Pestotnik SL, Lloyd JF et al. The epidemiology of nephrotoxicity associated with conventional amphotericin B therapy. Am J Med 2001; 111: 528-534

51. White MH, Bowden RA, Sandler ES et al. Randomized double-blind clinical trial of amphotericin B colloidal dispersion vs amphotericin $\mathrm{B}$ in the empirical treatment of fever and neutropenia. Clin Infect Dis 1998; 27: 296-302.

52. Walsh TJ, Finberg RW, Arndt C, et al. Liposomal amphotericin B for empirical therapy in patients with persistent fever and neutropenia: National Institute of Allergy and Infectious Diseases Mycoses Study Group. N Engl J Med 1999; 340: 764-771

53. Rossert J. Drug-induced acute interstitial nephritis. Kidney Int 2001; 60: 804-817

54. Handa SP. Drug-induced acute interstitial nephritis: report of 10 cases. CMAJ 1986; 135: 1278-1281

55. Rihal CS, Textor SC, Grill DE et al. Incidence and prognostic importance of acute renal failure after percutaneous coronary intervention. Circulation 2002; 105: 2259-2264

56. Dangas G, Iakovou I, Nokolsky E et al. Contrast-induced nephropathy after percutaneous coronary interventions in relation to chronic kidney disease and hemodynamic variables. Am J Cardiol 2005; 95: 13-19

57. Lindsay J, Apple S, Pinnow EE et al. Percutaneous coronary interventionassociated nephropathy foreshadows increased risk of late adverse events in patients with normal baseline serum creatinine. Catheter Cardiovasc Interv 2003; 59: 338-343
58. Freeman RV, O’Donnell M, Share D et al. Nephropathy requiring dialysis after percutaneous coronary intervention and the critical role of adjusted contrast dose. Am J Cardiol 2002; 90: 1068-1073

59. Mehran R, Aymong ED, Nikolsky E et al. A simple risk score for prediction of contrast induced nephropathy after percutaneous coronary intervention: Development and initial validation. J Am Coll Cardiol 2004; 44: 1393-1399

60. McCullough PA, Bertrand ME, Brinker JA et al. A meta-analysis of the renal safety of isosmolar iodixanol compared with low-osmolar contrast media. J Am Coll Cardiol 2006; 48: 692-699

61. Solomon R. The role of osmolality in the incidence of contrast-induced nephropathy: A systematic review of angiographic contrast media in high risk patients. Kidney Int 2005; 68: 22562263

62. Trivedi HS, Moore $\mathrm{H}$, Nasr $\mathrm{S}$ et al. A randomized prospective trial to assess the role of saline hydration on the development of contrast nephrotoxicity. Nephron Clin Pract 2003; 93: C29-C34

63. Mueller C, Buerkle G, Buettner HJ. Prevention of contrast media associated nephropathy: Randomized comparison of 2 hydration regimens in 1620 patients undergoing coronary angioplasty. Arch Intern Med 2002; 162: 329-336

64. Gonzales DA, Norsworthy KJ, Kern SJ et al. A meta-analysis of $\mathrm{N}$-acetylcysteine in contrast-induced nephrotoxicity: unsupervised clustering to resolve heterogeneity. BMC Med. 2007; 5: 32.

65. Hoffman U, Fischereder M, Kruger B et al. The value of $\mathrm{N}$-acetylcysteine in the prevention of radiocontrast agentinduced nephropathy seem questionable. J Am Soc Nephrol 2004; 15: 407-410

66. Bagshaw SM, Ghali WA. Theophylline for Prevention of Contrast-Induced Nephropathy- A Systematic Review and Meta-analysis. Arch Intern Med. 2005;165:1087-1093.

67. Stacul F, Adam A, Becker CR et al. Strategies to reduce the risk of contrastinduced nephropathy. Am J Cardiol 2006; 98: 59K-77K 
68. Marenzi G, Marana I, Lauri G, et al. The prevention of radiocontrast-agentinduced nephropathy by hemofiltration. N Eng J Med 2003; 349: 1333-1340

69. Brivet FG, Kleinknecht DJ, Loirat $P$ et al. Acute renal failure in intensive care units- causes, outcome, and prognostic factors of hospital mortality: A prospective multicenter study. French Study Group on Acute Renal Failure. Crit Care Med 1996; 24: 192-198

70. Guerin C, Girard R, Selli JM, et al. Initial versus delayed acute renal failure in the intensive care unit: A multicenter prospective epidemiological study. Rhone-Alpes Area Study Group on Acute Renal Failure. Am J Respir Crit Care Med 2000; 161: 872-879

71. Mehta RL, Pascual MT, Soroko S et al. Diuretics, mortality and nonrecovery of renal function in acute renal failure. JAMA 2002; 288: 2547-2553

72. Uchino S, Doig GS, Bellomo $\mathrm{R}$ et al. Diuretics and mortality in acute renal failure. Crit Care Med 2004; 32: 16691677

73. Ho KM, Sheridan DJ. Meta-analysis of frusemide to prevent or treat acute renal failure.BMJ 2006; 333: 420

74. Bagshaw SM, Delaney A, Haase M et al. Loop diuretics in the management of acute renal failure: A systematic review and meta-analysis. Crit Care Resusc 2007; 9: 68

75. Sampath S, Moran JL, Graham PL et al. The efficacy of loop diuretics in acute renal failure: Assessment using Bayesian evidence synthesis techniques. Crit Care Med 2007; 35: 2516-2524

76. Brunton P, Lazo J, Parker K, editors: Goodmans and Gillman's The Pharmacological Basis of Therapeutics $11^{\text {th }}$ ed. Milano McGraw Hill Publishing; 2005

77. Solomon R, Werner C, Mann D et al. Effect of saline, mannitol and furosemide to prevent acute decreases of renal function induced by radiocontrast agents. New Engl J Med 1994; 331: 1416-1420

78. Homsi E, Barreiro MF, Orlando JM et al. Prophylaxis of acute renal failure in patients with rhabdomyolysis. Ren Fail 1997; 19: 283-288

79. Griesdale DE, de Souza RJ, van Dam RM et al. Intensive insulin therapy and mortality among critically ill patients: a meta-analysis including NICE-SUGAR study data. CMAJ, 2009; 180: 821-827

80. Schetz M, Vanhorebeek I, Wouters PJ et al. Tight blood glucose control is renoprotective in critically ill patients. J Am Soc Nephrol. 2008; 19: 571-8. 